\title{
MESURES RESTRICTIVES DE L'UNION EUROPÉENNE POUR LUTTER CONTRE LE TERRORISME, DROITS DE LA DÉFENSE ET JURISPRUDENCE DE LA COUR DE JUSTICE DE L'UNION EUROPÉENNE
}

DOI: $10.47743 /$ rdc-2016-4-0003

Diégo COLAS

Brice FODDA ${ }^{2}$

\section{Résumé}

Dans son arrêt du 3 septembre 2008, Kadi et Al Barakaat International Foundation/Conseil et Commission (C-402/05 P, dit "arrêt Kadi I", point 81), la Cour de Justice de I'Union Européenne a souligné avec une certaine solennité que «la Communauté Européenne est une communauté de droit en ce que ni ses États membres ni ses institutions n'échappent au contrôle de la conformité de leurs actes à la charte constitutionnelle de base qu'est le traité de l'Union».

Dans le cadre de la même affaire, quelques années plus tard, dans son arrêt $d u$ 18 juillet 2013, Commission, Conseil et Royaume-Uni/Kadi (C-584/10 P, C-593/10 P et C-595/10 P, dit "arrêt Kadi II", point 66), la Cour de Justice de I'Union Européenne a de nouveau qualifié de "constitutionnelle» la garantie qu'incarne, dans une Union de droit, le contrôle juridictionnel de la légalité de tout acte de l'Union, y compris de ceux qui, comme en l'occurrence, mettent en œuvre un acte de droit international, au regard des droits fondamentaux garantis par l'Union.

Ainsi, c'est à l'occasion de la lutte contre le terrorisme qu'a été rappelée et consacrée une caractéristique qui, certes, existait déjà en droit de l'Union ${ }^{3}$ et qui permettait à l'Union Européenne de considérer son traité fondateur comme un acte qui n'était pas seulement de nature contractuelle ou synallagmatique, mais comme véritablement constitutionnel, c'est-à-dire fondateur d'un ordre juridique construit sur des valeurs partagées.

\footnotetext{
${ }^{1}$ Diégo Colas est directeur adjoint des affaires juridiques du Ministère des affaires étrangères et du développement international du gouvernement français.

${ }^{2}$ Brice Fodda est rédacteur dans cette direction. Ils sont agents du gouvernement français devant la Cour de justice de l'Union Européenne. Les propos tenus dans cet article sont exprimés à titre personnel et n'engagent pas le gouvernement français.

${ }^{3}$ Voir arrêt de la Cour du 23 avril 1986, Les Verts/Parlement européen (294/83, point 23) où la qualification du traité comme «charte constitutionnelle de base» a permis de juger recevable le recours d'un parti politique contre un acte du Parlement européen.
} 
II n'est guère surprenant que la lutte contre le terrorisme soit un domaine dans lequel il est particulièrement pertinent de regarder le traité de l'Union comme un acte constitutionnel. En effet, cette politique de lutte contre le terrorisme constitue un domaine où se rencontrent des logiques proprement constitutionnelles: articulation entre l'ordre juridique qu'est l'Union et le droit international; nécessité de préserver les droits fondamentaux, en particulier les droits de la défense et le droit à un contrôle juridictionnel.

Le présent article s'attachera d'abord à cerner la notion de "mesures restrictives» en droit de l'Union et à examiner les applications de cette notion dans le cadre de la lutte contre le terrorisme (A). II analysera ensuite les moyens par lesquels a été assuré l'équilibre entre l'efficacité de la politique de l'Union de lutte contre le terrorisme et la protection des droits fondamentaux, en distinguant selon que cette politique s'inscrit dans le cadre plus large d'une coopération internationale (B) ou qu'il s'agit d'une politique autonome de I'Union (C).

Mots-clés: la lutte contre le terrorisme; droit de l'Union; charte constitutionnelle

\section{A. Les mesures restrictives en droit de l'Union}

Les mesures restrictives désignent en droit de I'Union ce qui s'appelle souvent «sanctions économiques» dans le langage courant. Elles figurent parmi les outils dont I'Union Européenne dispose afin de promouvoir les objectifs de sa politique étrangère et de sécurité commune, à savoir la paix, la démocratie ainsi que le respect de l'État de droit, des droits de l'homme et du droit international. L'objectif du recours aux mesures restrictives est d'influencer les comportements en évitant le recours à la force.

Certains régimes de mesures restrictives ont pour finalité de lutter contre le terrorisme. D'autres régimes poursuivent d'autres finalités: la lutte contre la prolifération nucléaire par l'Iran, l'évolution de certains pays tiers considérée comme contraire aux objectifs de I'Union (Syrie, Zimbabwe, Corée du Nord) ou encore la nécessité d'aider certains gouvernements de pays tiers issus de révolte populaires à recouvrer des biens spoliés par les anciens dirigeants (Tunisie, Égypte, Ukraine).

Il convient donc d'emblée de bien distinguer deux situations où le terrorisme peut être invoqué pour justifier l'imposition d'un régime de mesures restrictives. D'une part, il existe des régimes de mesures restrictives par lesquels l'Union désigne certaines personnes ou certaines entités telles que des fondations, des associations ou des mouvements sans personnalité morale, au motif que ces personnes ou entités sont elles-mêmes engagées dans des activités de terrorisme. Il en résulte, pour les personnes et entités concernées, des restrictions importantes telles que le gel de leurs avoirs dans I'Union ou une interdiction de se rendre dans l'Union. D'autre part, il existe des régimes 
Mesures restrictives de l'Union Européenne pour lutter contre le terrorisme...

de mesures restrictives qui visent à modifier la politique étrangère de certains pays tiers, y compris dans la mesure où ces derniers soutiennent certains mouvements terroristes. Un tel régime conduirait au gel d'avoirs et à des interdictions de voyager à l'encontre de certaines catégories de population qui dirigent le pays concerné ou qui le soutiennent activement.

Qu'il s'agisse des régimes de lutte contre le terrorisme ou de ceux visant à modifier le comportement d'un pays tiers, ces régimes de mesures restrictives peuvent relever d'une logique de coopération ou d'autonomie. Ainsi, certains régimes s'inscrivent dans l'application par l'Union d'un régime international adopté sur le fondement du chapitre VII de la Charte des Nations Unies, et en particulier son article 41. D'autres sont des régimes autonomes de l'Union Européenne, adoptés par elle seule au titre de sa politique étrangère.

Dans les deux cas, il revient au Conseil de l'Union Européenne d'adopter les mesures restrictives au moyen d'une décision prise sur le fondement d'un article du traité relatif à la politique étrangère et de sécurité commune, par un vote à l'unanimité. Une telle décision peut prévoir des mesures qui relèvent de la compétence des États membres, telles que des interdictions de se rendre dans l'Union (interdiction d'entrée sur le territoire, refus de visa). Le plus souvent, elle prévoit aussi des mesures qui relèvent de la compétence de l'Union, telles qu'une mesure de gels d'avoirs qui concerne les fonds et ressources économiques détenus ou contrôlés par les personnes et entités désignées. Pour mettre en œuvre une telle mesure, un acte législatif de l'Union est alors nécessaire. II prend la forme d'un règlement de l'Union adopté sur le fondement de l'article 215 TFUE.

On retrouve cette distinction entre régime dit onusien et régime dit autonome, ainsi que cette articulation entre une décision adoptée sur le fondement de la politique étrangère et de sécurité commune, d'une part, et un règlement de l'Union adoptée sur le fondement de l'article 215 TFUE, d'autre part, en ce qui concerne la lutte contre le terrorisme. Ainsi, les sanctions mises en œuvre par l'Union Européenne pour combattre le terrorisme reposent à ce jour sur deux régimes:

- un régime est dédié à la transposition, au niveau européen, des mesures prises par le comité des sanctions contre Al Qaïda, récemment élargi à Daesh, créé par la résolution 1267 (1999) du Conseil de sécurité des Nations Unies. Ainsi, ce régime applique en droit de l'Union les désignations imposées par cette résolution par le biais de la position commune 2002/402/PESC du 27 mai 2002, abrogée et remplacée par la décision 2016/1693 du 20 septembre 2016, et du règlement (CE) $n^{\circ}$ 881/2002 du 27 mai 2002. II comprend aujourd'hui des mesures de gels d'avoirs et d'interdictions de voyager, ainsi que l'interdiction de fournir, vendre, transférer ou exporter, directement ou indirectement, certains biens (notamment liés à des activités militaires) et services aux personnes et entités désignées;

- un régime de sanctions autonome a été, à l'origine, créé pour mettre en œuvre l'obligation générale de lutter contre le terrorisme qui résulte de la résolution 1373

STUDIES AND ARTICLES 
(2001) adoptée par le Conseil de sécurité des Nations Unies à la suite des attentats du 11 septembre 2001. II repose sur la position commune 2001/931/PESC du 27 décembre 2001, et le règlement (CE) $n^{\circ}$ 2580/2001 du 27 décembre 2001. Dans le cadre de ce régime qui prévoit des mesures de gels d'avoirs, la désignation d'un individu ou d'un groupe est conditionnée à l'existence préalable d'une décision prise par une autorité nationale compétente (judiciaire ou administrative) pour des faits liés au terrorisme.

Avant d'examiner chacun de ces régimes, il convient de relever que, si ces régimes visent à produire des incitations pour que certaines personnes ou entités situées hors du territoire de l'Union modifient leur comportement, l'Union s'attache à éviter que ses décisions aient, en droit, un effet extraterritorial illicite en droit international, c'est-àdire dénué d'un lien suffisant avec son territoire. C'est ainsi que chaque règlement de I'Union prévoyant des mesures restrictives comprend un article qui précise que le règlement s'applique sur le territoire de l'Union, dans les aéronefs ou navires de I'Union, à des personnes morales de l'Union, y compris leurs succursales mais pas leurs filiales de droit d'un pays tiers, ou aux transactions commerciales se déroulant dans I'Union ${ }^{4}$. Sur ce point, les sanctions de l'Union se distinguent des sanctions américaines qui ont parfois tendance à négliger la nécessité, issue du droit international, de prévoir un lien suffisant entre une mesure adoptée par les États-Unis et le territoire de ce pays puisque certains sanctions, dites «secondaires», s'appliquent à des opérateurs non américains hors de tout lien avec le territoire américain et que d'autres, dites "primaires», donc reposant en théorie sur un lien avec le territoire américain, se contentent parfois d'un lien extrêmement ténu, tel que l'existence de $10 \%$ des composantes d'un produit qui serait d'origine américaine.

Il convient à présent d'examiner la façon dont le juge de l'Union a veillé à préserver l'efficacité des régimes de l'Union tout en assurant le respect des droits fondamentaux consacrés par le traité sur l'Union Européenne. En effet, le juge de l'Union est compétent pour juger un recours en annulation formé par les personnes ou entités désignées, même si elles n'ont pas la personnalité morale ${ }^{5}$, en vue de contrôler la légalité des mesures restrictives de l'Union Européenne à l'encontre de personnes physiques ou morales, conformément à l'article 275 , second alinéa, TFUE.

Pour partie, l'équilibre recherché par le juge de l'Union a reposé sur l'application d'une méthode commune à l'ensemble des régimes de mesures restrictives. L'Union est

\footnotetext{
${ }^{4}$ Cette pratique a été recommandée par les lignes directrices concernant la mise en œuvre et l'évaluation des mesures restrictives du Conseil du 15 juin 2012.

${ }^{5}$ Voir arrêt du 18 janvier 2007, PKK et KNK/Conseil, C-229/05 P, point 114: Les dispositions du statut de la Cour de justice n'ont pas été conçues en vue de l'introduction de recours par des organisations n'ayant pas la personnalité juridique. Dans la situation exceptionnelle [d'un recours d'une telle organisation contre sa désignation dans un régime de mesures restrictives], les règles procédurales doivent être appliquées en les adaptant aux circonstances de l'espèce dans la mesure nécessaire. Il s'agit d'éviter un formalisme excessif qui reviendrait à nier toute possibilité d'agir en annulation alors même que l'entité en question a fait l'objet de mesures restrictives.
} 
Mesures restrictives de l'Union Européenne pour lutter contre le terrorisme...

tenue d'adopter, pour chaque régime, des critères de désignation adaptés. Ensuite, pour chaque désignation, le juge de l'Union vérifie que l'Union a formulé une motivation suffisante, rappelant le critère auquel la désignation se rattache, mais aussi les motifs précis et concrets qui permettent ce rattachement. Le juge de l'Union contrôle aussi le bien-fondé de la désignation, en vérifiant les éléments sur lesquels I'Union s'est basée. II contrôle précisément le respect des garanties procédurales. II est souvent amené à se prononcer également sur la proportionnalité des mesures prises à l'encontre des personnes désignées.

Ainsi, c'est en appliquant une méthode à peu près commune à tous les régimes de mesures de restrictives que le juge procède au contrôle de légalité d'un acte de l'Union, afin de veiller au respect de la Charte constitutionnelle de base qu'est le traité, y compris en ce qu'il garantit les droits fondamentaux.

Cependant, du fait des particularités des deux régimes de mesures restrictives de I'Union Européenne contre le terrorisme, l'équilibre recherché par le juge de l'Union fait aussi apparaître des problématiques propres à chacun de ces régimes.

\section{B. Le régime onusien de mesures restrictives de l'Union}

\section{pour lutter contre le terrorisme}

Le régime de mesures restrictives régi par la position commune 2002/402/PESC du 27 mai 2002, abrogée et remplacée par la décision 2016/1693 du 20 septembre 2016, et par le règlement $(C E) n^{\circ} 881 / 2002$ du 27 mai 2002 vise à mettre en œuvre dans l'Union Européenne les obligations qui découlent de la résolution 1267 (1999) du Conseil de sécurité des Nations Unies.

Ainsi, il présente deux caractéristiques que n'ont pas les régimes autonomes de mesures restrictives de l'Union Européenne, et qui auraient pu avoir une incidence sur l'étendue du contrôle juridictionnel auquel procède le juge de l'Union. Tel est ce que plaidaient le Conseil, la Commission, plusieurs États membres dans les affaires Kadi I et II, et tel est aussi ce que concluait l'avocat général dans ses conclusions sous l'affaire Kadi II.

Une première caractéristique du régime onusien de lutte contre le terrorisme de I'Union est qu'il existe une obligation pour l'Union et pour ses États membres de mettre en œuvre la résolution 1267 du Conseil de sécurité des Nations Unies. En outre, s'agissant des États membres, l'article 103 de la Charte des Nations Unies dispose que, en cas de conflit entre les obligations des membres des Nations Unies en vertu de la présente Charte et leurs obligations en vertu de tout autre accord international, les premières prévaudront.

Ainsi, il se pose la question de savoir si le droit de l'Union est même susceptible de faire obstacle à l'application de la Charte des Nations Unies puisque, en cas d'éventuel conflit, celle-ci prévaut sur toute obligation en vertu d'un autre accord international. 


\section{Diégo COLAS • Brice FODDA}

C'est dans ce contexte que, comme il a été indiqué au début de cet article, la Cour de Justice de l'Union Européenne a rappelé, dans son arrêt Kadi I, que le traité de l'Union n'était pas simplement «un autre accord international» mais représentait «la charte constitutionnelle de base» de l'ordre juridique que constitue l'Union Européenne. Ainsi, les États membres (et les institutions européennes) ne pouvaient pas faire fi des obligations qui découlent des traités de I'Union, notamment des droits fondamentaux.

C'est ainsi que la Cour a jugé, dans l'arrêt Kadi I, que, même si elle est effectuée en application d'une résolution des Nations Unies, la désignation d'une personne ou entité au titre du régime de mesures restrictives de l'Union doit faire l'objet d'un contrôle «en principe complet» (et non pas d'un contrôle restreint réduit à une vérification de la plausibilité des motifs invoqués par l'Union). La Cour a précisé, dans l'arrêt Kadi II, que ce contrôle en principe complet porte sur la motivation et sur le bien-fondé de la désignation.

Une seconde caractéristique du régime onusien de lutte contre le terrorisme de I'Union est qu'il peut apparaître plus logique que le mécanisme de révision des désignations soit effectué au niveau des Nations Unies plutôt que dans les ordres juridiques internes des membres de l'Union, y compris celui de l'Union Européenne.

C'est ainsi que, depuis plusieurs années, le Conseil de sécurité des Nations Unies a mis en place, dans l'ensemble des régimes de sanctions qu'il adopte, des dispositifs visant à améliorer la mise en œuvre et la motivation des sanctions et à permettre aux entités et personnes sanctionnées de contester leur désignation. En particulier, le Conseil de Sécurité décide de plus en plus souvent de créer un comité des sanctions, organe subsidiaire du Conseil réunissant tous les États membres et dont les trois fonctions principales sont d'actualiser les listes de sanctions (désignation et radiation des personnes et entités), de surveiller leur mise en œuvre et d'en clarifier les modalités d'application.

Par ailleurs, en matière de garanties procédurales, la résolution 1730 du 19 décembre 2006 a mis en œuvre pour l'ensemble des régimes de sanctions une procédure de radiation des personnes désignées. Ainsi, pour chaque régime de sanctions, un «point focal» est chargé de recueillir les demandes de radiation et de les transmettre au comité des sanctions compétent, aux gouvernements à l'origine de l'inscription sur la liste ainsi qu'au gouvernement de l'État de nationalité et de l'État de résidence de l'intéressé. Ce point focal informe ensuite le demandeur de la décision du comité.

Pour ce qui concerne le régime de sanctions contre Al-Qaïda, récemment élargi à Daesh, ces garanties procédurales ont été renforcées, par la résolution 1904 du 18 décembre 2009 qui a créé une fonction de Médiateur (Ombudsperson), se substituant au point focal. Ce Médiateur a pour mission de recevoir les demandes de radiation, de les instruire et de transmettre un rapport au comité des sanctions sur la demande de radiation. Le Médiateur recommande soit le maintien de l'individu ou de l'entité sur la liste, soit sa radiation. 
Mesures restrictives de I'Union Européenne pour lutter contre le terrorisme...

Cependant, dans l'arrêt Kadi II, la Cour a bien précisé que l'existence de ces mécanismes n'est pas de nature à justifier une atténuation du contrôle auquel doit procéder le juge de l'Union. En effet, la Cour de Justice de l'Union Européenne a jugé que, en dépit des améliorations que constituent la création du point focal ou du Médiateur, les procédures de radiation et de révision d'office instituées au niveau des Nations Unies n'offrent pas aux personnes concernées les garanties d'une protection juridictionnelle effective. II s'en déduit qu'un éventuel nouveau renforcement de ce type de garanties au sein des Nations Unies ne changerait pas cette conclusion, et que seule une véritable transformation en profondeur de ces mécanismes pour en faire un mécanisme juridictionnel aurait un tel effet.

II résulte de ce qui précède que, malgré ces deux caractéristiques, il apparaît bien que les actes de l'Union, même pris en application stricte de résolutions du Conseil de sécurité, font l'objet d'un contrôle juridictionnel normal au regard en particulier du respect des droits fondamentaux. Ainsi, l'autorité compétente de l'Union doit communiquer à l'intéressé les éléments sous-tendant sa décision, doit permettre à la personne concernée de faire connaître utilement son point de vue à l'égard des motifs retenus à son encontre et doit examiner, à la lumière des observations faites par l'intéressé, le bien-fondé de ces motifs. En cas de besoin, il incombe à cette autorité de solliciter la collaboration du comité des sanctions et, à travers ce dernier, de l'État membre de l'ONU ayant proposé l'inscription de la personne concernée sur la liste récapitulative, pour obtenir la communication d'informations ou d'éléments de preuve lui permettant de procéder à un examen soigneux et impartial du bien-fondé des motifs en question.

C'est dans le cadre de l'examen des affaires Kadi que s'est posée une question qui dépasse celle de la lutte contre le terrorisme dans un cadre onusien, car elle se pose aussi dans le cadre des régimes de mesures restrictives à l'encontre du comportement de certains pays ${ }^{6}$, qui est la difficulté de soumettre à une procédure juridictionnelle les éléments sur lesquels le Conseil de l'Union s'est fondé pour considérer qu'une personne doit être désignée au titre d'un régime de mesures restrictives.

En effet, il n'est pas exceptionnel que ces éléments soient de nature à révéler les sources, les modes opératoires ou les techniques de coopération qui permettent aux services de renseignement d'effectuer leurs missions. Or, dans le cadre d'une procédure juridictionnelle, ces éléments font l'objet d'un examen contradictoire, ce qui suppose que la personne faisant l'objet des mesures restrictives, y ait accès. Cette difficulté était mise en avant par ceux qui estimaient que l'exigence d'un contrôle juridictionnel normal sur le bien-fondé des désignations de personnes et d'entités dans un régime de mesures restrictives aboutirait en pratique à une impossibilité de mettre en œuvre ce régime.

\footnotetext{
${ }^{6}$ On verra infra que cette problématique ne se pose pas dans le cadre du régime de mesures restrictive autonome de l'Union contre le terrorisme, pour lequel une toute autre solution a été adoptée par l'Union.
}

STUDIES AND ARTICLES 


\section{Diégo COLAS • Brice FODDA}

En réponse, la Cour a admis que des considérations impérieuses touchant à la sûreté de l'Union ou de ses États membres ou à la conduite de leurs relations internationales puissent s'opposer à la communication de certaines informations ou de certains éléments de preuve à la personne concernée. Cependant, elle a ajouté que, en pareil cas, il incombe au juge de l'Union, auquel ne saurait être opposé le secret ou la confidentialité de ces informations ou éléments, de mettre en œuvre, dans le cadre du contrôle juridictionnel qu'il exerce, des techniques permettant de concilier, d'une part, les considérations légitimes de sécurité quant à la nature et aux sources de renseignements ayant été pris en considération pour l'adoption de l'acte concerné et, d'autre part, la nécessité de garantir à suffisance au justiciable le respect de ses droits procéduraux, tels que le droit d'être entendu ainsi que le principe du contradictoire (point 125 de l'arrêt Kadi II).

C'est pour répondre à cette considération fondamentale de l'arrêt Kadi II, et en se conformant étroitement aux indications fournies dans cet arrêt, que les règlements de procédure de la Cour et du Tribunal ont été modifiés pour inclure une procédure relative à l'examen de telles pièces, qui vise à concilier les considérations légitimes de sécurité de l'Union et de ses États membres, d'une part, et la nécessité de garantir à suffisance au justiciable le respect de ses droits procéduraux, d'autre part.

Ainsi, le règlement de procédure du Tribunal inclut à présent un article 105 relatif au traitement des renseignements ou pièces touchant à la sûreté de l'Union ou à celle d'un ou de plusieurs de ses États membres ou à la conduite de leurs relations internationales.

Cet article prévoit la possibilité pour une partie au principal de fonder ses prétentions sur des renseignements dont la divulgation porterait atteinte à la sûreté de I'Union ou à celle de l'un ou plusieurs de ses États membres ou à la conduite de leurs relations internationales. En pratique, on voit mal la personne ou l'entité désignée être en mesure de démontrer qu'elle entend fonder sa demande d'annulation de la désignation dont elle fait l'objet sur de tels éléments, et encore moins que des éléments susceptibles de porter atteinte à la sûreté de l'Union ou à celle de l'un ou plusieurs de ses États membres ou à la conduite de leurs relations internationales ne puissent pas être communiqués aux institutions de l'Union ou aux États membres. Par conséquent, ce sont principalement le Conseil ou la Commission, selon le régime de mesures restrictives en cause, c'est-à-dire l'institution ayant procédé à la désignation, qui est susceptible d'invoquer cette procédure.

La procédure vise à maintenir un équilibre entre les considérations légitimes de sécurité de l'Union et de ses États membres, d'une part, et la nécessité de garantir à suffisance au justiciable le respect de ses droits procéduraux, d'autre part. Ainsi, la partie qui entend se fonder sur de tels éléments doit transmettre les éléments et une demande de traitement confidentiel au Tribunal. Celui-ci doit d'abord évaluer si les éléments en cause sont pertinents et s'il est convaincu de la nécessité de leur conserver un caractère confidentiel.

CONSTITUTIONAL LAW REVIEW 
Mesures restrictives de I'Union Européenne pour lutter contre le terrorisme...

Si le Tribunal décide que lesdits éléments sont effectivement pertinents mais qu'il n'est pas justifié de leur conférer un caractère confidentiel, il donne le choix à la partie principale qui a transmis les éléments soit d'autoriser leur transmission à l'autre partie, soit de les retirer, de sorte que le Tribunal ne pourra fonder sa décision sur ces éléments dans son arrêt. En revanche, si le Tribunal décide que lesdits éléments sont effectivement pertinents et qu'il est justifié de leur conférer un caractère confidentiel, il procède à une mise en balance des exigences liées au droit à une protection juridictionnelle effective et de celles découlant de la sûreté de l'Union ou de ses États membres et, par ordonnance motivée, précise les modalités permettant la meilleure conciliation possible entre ces exigences.

II peut s'agir, comme le précise expressément l'article 105, paragraphe 6, du règlement de procédure du Tribunal, de demander à la partie principale ayant invoqué les éléments concernés de produire une version non confidentielle ou un résumé non confidentiel des éléments en question, comportant leur contenu essentiel et permettant à l'autre partie, dans la plus large mesure possible, de faire valoir ses observations. En outre, les éléments en cause seront conservés dans un local dédié et apte à la protection et à la consultation dans des conditions optimales de sécurité de pièces d'un tel niveau de confidentialité. Enfin, lorsque le Tribunal statue sur le litige, il peut, par dérogation au principe du contradictoire et en se limitant à ce qui est strictement nécessaire, fonder son jugement sur ces éléments, mais il doit, ce faisant, tenir compte de ce que l'autre partie n'a pas pu faire valoir ses observations sur les éléments eux-mêmes.

Dans l'hypothèse où l'arrêt rendu par le Tribunal ferait l'objet d'un pourvoi devant la Cour, l'article 190 bis du règlement de procédure de la Cour, relatif au traitement des renseignements ou pièces produits devant le Tribunal au titre de l'article 105 de son règlement de procédure, permet à la Cour de prendre connaissance des éléments en question tout en maintenant la protection due à leur caractère confidentiel.

L'application de cette procédure dépendait de l'adoption par la Cour et par le Tribunal de règles de sécurité spécifiques, ainsi que de la mise en place concrète du local où les éléments en cause seront conservés et consultés. Ces conditions étant remplies, il est prévu que cette application débute effectivement en janvier 2017.

Ainsi, le droit de l'Union a été amené, dans la mise en œuvre du régime onusien de lutte contre le terrorisme de l'Union, à préciser l'articulation entre la Charte des Nations Unies et le respect des droits fondamentaux dans l'Union Européenne, ainsi qu'entre les garanties fondamentales prévues dans le dispositif onusien et les mécanismes de contrôle juridictionnels en droit de l'Union. Il a aussi été amené à préciser comment prendre en compte de façon équilibrée, dans la procédure devant le juge de l'Union, les exigences de la sûreté de l'Union et de ses États membres et celles liées au droit à une 
protection juridictionnelle effective. Rarement aura-t-il été aussi pertinent de conférer au traité de l'Union une valeur constitutionnelle, c'est-à-dire fondée sur une obligation particulièrement exigeante de respecter les valeurs qui sous-tendent la construction européenne.

\section{Le régime autonome de mesures restrictives de l'Union}

\section{pour lutter contre le terrorisme}

C'est sur un équilibre un peu différent que repose le régime établi par la position commune 2001/931/PESC du 27 décembre 2001, et par le règlement (CE) $n^{\circ}$ 2580/2001 du 27 décembre 2001. II en résulte que cette obligation particulièrement exigeante de respecter les valeurs qui sous-tendent la construction européenne prend, pour ce régime, un aspect un peu moins spectaculaire.

Ce régime est l'un des plus anciens régimes de mesures restrictives de l'Union. C'est peut-être ce qui explique sa structure inhabituelle. En effet, ce régime prévoit d'abord l'adoption d'une décision nationale désignant une entité comme terroriste et adoptée dans le respect du principe du contradictoire, tel qu'il est mis en œuvre dans chaque État membre. Alors que, en 2001, l'Union, et notamment le juge de l'Union, n'avait guère été appelée à agir dans une matière où les implications de sécurité étaient considérables, il pouvait paraître de bonne politique de se fonder sur des mécanismes éprouvés, et d'ailleurs pas nécessairement identiques ou reproductibles d'un État membre à un autre ${ }^{7}$, pour assurer la conciliation entre respect des droits de la défense et lutte contre le terrorisme.

Il en résulte que, dans son contrôle, le juge de l'Union n'a pas eu besoin de recourir au vocabulaire constitutionnel qui retient l'attention dans les arrêts Kadi I et II. Plus généralement, le contrôle juridictionnel de ce régime a été globalement moins spectaculaire, du moins jusqu'à l'annulation récente de l'inscription sur la liste européenne des organisations terroristes des Tigres Tamouls et du Hamas ${ }^{8}$, et encore cette annulation avait-elle surtout des motifs procéduraux sur lesquels nous revenons infra.

Peu à peu, cependant, le juge de l'Union a été appelé à veiller au respect des droits fondamentaux et à préciser dans quelles conditions il est possible de se fonder sur une décision nationale pour procéder à la désignation d'une personne ou entité sur une liste européenne d'organisation terroriste.

\footnotetext{
${ }^{7}$ On peut penser, par exemple, au mécanisme des avocats spéciaux qui existe au Royaume-Uni (voir arrêt de la Cour du 4 juin 2013, ZZ, C-300/11) et qu'il n'est pas nécessairement envisageable de reproduire dans d'autres États membres ou au niveau de l'Union, faute d'une organisation de la profession comparable à ce qui existe au Royaume-Uni.

${ }^{8}$ Voir arrêts du Tribunal du 16 octobre 2014, LTTE/Conseil (T-208/11 et T-508/11), et du 17 décembre 2014, Hamas/Conseil (T-400/10).
} 
Mesures restrictives de I'Union Européenne pour lutter contre le terrorisme...

Tout d'abord, le juge de l'Union a apporté plusieurs précisions sur le type de décisions nationales qui était admissible. En effet, la règlementation de l'Union précise que cette décision doit être prise par une autorité compétente, doit faire suite à une procédure d'enquête, de poursuite ou de condamnation et doit être fondée sur des preuves et indices sérieux et crédibles, et enfin que le Conseil doit avoir donné des raisons spécifiques et concrètes pour lesquelles il considère que la désignation sur la liste européenne de la personne ou de l'entité est justifiée.

A cet égard, le juge de l'Union a précisé que la position commune 2001/931 ne requiert pas que la décision de l'autorité compétente s'inscrive dans le cadre d'une procédure pénale stricto sensu. Ainsi, la décision nationale en cause peut aussi être prise dans le cadre d'une procédure ayant pour objet des mesures de type préventif (voir arrêt du Tribunal du 9 septembre 2010, Al-Aqsa/Conseil, T-348/07, confirmé par la Cour dans son arrêt du 15 novembre 2012, Al-Aqsa/Conseil, C-539/10 P et C-550/10 P). II en résulte que la décision nationale en cause peut être adoptée par une autorité administrative, comme c'est le cas d'un proscription order au Royaume-Uni ou d'un Sanctieregeling terrorisme (arrêté de sanctions en matière de terrorisme) aux Pays-Bas.

En revanche, le Tribunal a jugé que, pour pouvoir être valablement invoquée par le Conseil, une décision d' "ouverture d'enquêtes ou de poursuites» doit s'inscrire dans le cadre d'une procédure nationale visant à titre principal à l'imposition d'une mesure de type préventif ou répressif à l'encontre de l'intéressé, au titre de la lutte contre le terrorisme. Il a ainsi jugé que cette exigence n'est pas remplie par une décision d'une autorité judiciaire nationale qui concerne une contestation portant sur des droits et des obligations de caractère civil et qui ne se prononcerait qu'à titre accessoire et incident sur l'implication possible de l'intéressé dans une telle activité (arrêt du 30 septembre 2009, Sison/Conseil, T-341/07).

Ensuite, le juge de l'Union a également été confronté au cas où les décisions «nationales» invoquées pour justifier une désignation sur la liste européenne des organisations terroristes étaient des décisions de pays tiers. Ainsi, dans l'affaire LTTE/Conseil, précitée, le Conseil invoquait deux décisions nationales, I'une britannique, l'autre indienne. Dans l'affaire Hamas/Conseil, précitée, il invoquait deux décisions nationales, l'une britannique, l'autre américaine.

Dans l'arrêt LTTE/Conseil, le Tribunal a admis la possibilité de se fonder sur une décision indienne ${ }^{9}$ à condition de démontrer que la procédure dans le pays tiers concerné respecte les principes de l'État de droit et notamment le principe du contradictoire. Dans ses conclusions dans le pourvoi (toujours pendant) du Conseil contre cet arrêt, l'avocat général Mme Sharpston propose de confirmer cette solution, qui est effectivement raisonnable mais qui présente une difficulté pratique. En effet, s'il

\footnotetext{
${ }^{9}$ Il est curieux de constater que, dans l'arrêt Hamas/Conseil, précité, le Tribunal fait référence à la décision américaine (notamment au point 97) sans se demander s'il pouvait tenir compte d'une telle décision, et dans quelles conditions, alors qu'il avait longuement examiné cette question pour la décision indienne dans l'arrêt LTTE/Conseil, précité.
} 


\section{Diégo COLAS • Brice FODDA}

est légitime d'exiger que le Conseil vérifie que la décision nationale d'un pays tiers respecte les principes de l'État de droit et notamment le principe du contradictoire avant d'en tenir compte, il est délicat pour le Conseil de rendre public, dans sa décision de désignation, le résultat d'une telle vérification. En effet, les pays tiers pourraient s'émouvoir de ce que le Conseil prétende ainsi distribuer des bons et des mauvais points sur le respect de l'État de droit. L'arrêt de la Cour, encore à venir, sera donc intéressant sur la question de la motivation, davantage que sur celle de l'existence même de la condition qui, en soi, n'apparaît guère contestable.

Enfin, le juge de l'Union a précisé les vérifications auxquelles le Conseil doit procéder avant de renouveler l'inscription d'une personne ou une entité sur la liste européenne des organisations terroristes sur la base d'une décision nationale ancienne.

En effet, il est une garantie fondamentale pour les intéressés que la décision initiale d'inscription par l'Union soit réexaminée à échéances régulières. Lors de ce réexamen périodique, il incombe au Conseil de l'Union de s'assurer que l'inscription initiale reste justifiée. La question est donc de vérifier si, depuis l'inscription en cause ou depuis le réexamen précédent, la situation factuelle a changé de telle manière qu'elle ne permet plus de tirer la même conclusion concernant l'implication de la personne en question dans des activités terroristes (voir arrêt de la Cour Al Aqsa/Conseil, précité, point 82).

Pour cela, deux vérifications sont nécessaires.

Premièrement, le Conseil de l'Union doit s'assurer que l'appréciation initiale qui a fondé la décision nationale n'a pas été remise en cause. Ainsi, il s'agit de vérifier, formellement, que la décision est toujours en vigueur ${ }^{10}$ et quelles ont été les suites réservées à d'éventuels recours contre cette décision. Cette vérification permet d'établir que les preuves et indices qui avaient amené à considérer que la personne ou l'entité en cause se livrait à des activités terroristes n'ont pas été remis en cause.

Deuxièmement, il convient de vérifier que l'appréciation sur la base de laquelle la décision nationale a été prise continue de fonder la conclusion qu'une désignation de la personne ou de l'entité en cause est justifiée. La question peut s'avérer délicate lorsque la décision nationale devient ancienne avec le passage du temps, surtout si les circonstances politiques ont changé de façon substantielle, comme cela a pu être le cas avec la fin de la guerre civile au Sri Lanka. En présence d'un changement très important, il est possible que la désignation d'une personne ou entité sur la liste européenne des organisations terroristes reste justifiée mais qu'elle doive se fonder sur une nouvelle décision nationale, plus récente, parce que l'ancienne repose sur une appréciation qui n'est plus pertinente.

C'est sur ce point que portent les pourvois du Conseil contre les arrêts du Tribunal ayant annulé la désignation des Tigres Tamouls et du Hamas. Dans les affaires pendantes Conseil/LTTE (C-599/14 P) et Conseil/Hamas (C-79/15 P), le débat se

\footnotetext{
10 Ou qu'elle n'a été abrogée que parce que les autorités nationales considèrent que la décision européenne l'a remplacée, voir arrêt de la Cour Stichting Al Aqsa/Conseil, point 83.
}

CONSTITUTIONAL LAW REVIEW 
Mesures restrictives de l'Union Européenne pour lutter contre le terrorisme...

concentre sur ce que doit faire le Conseil lorsqu'il souhaite renouveler une décision d'inscription mais que la décision d'inscription est ancienne (2009 pour les Tigres Tamouls, 2003 pour le Hamas). Le Conseil peut-il se fonder uniquement sur cette décision ancienne ou peut-il invoquer des éléments nouveaux, tels que des attentats dont l'existence serait établie à partir de sources publiques et qui n'auraient pas fait l'objet d'une discussion contradictoire mais qui ont été revendiqués par l'entité, ce qui peut passer pour une forme de reconnaissance des faits?

En première instance, le Tribunal de l'Union Européenne avait annulé l'inscription de ces deux entités en considérant que le Conseil ne pouvait pas se fonder sur des éléments nouveaux qu'il aurait établi lui-même.

Le Conseil de l'Union a formé un pourvoi contre ces arrêts. Dans ses conclusions du 22 septembre 2016, l'avocat général conclut que l'arrêt du Tribunal doit être annulé. Selon lui, certes le Tribunal avait raison de considérer que le Conseil ne pouvait pas se fonder sur des éléments nouveaux qu'il aurait établi lui-même. Toutefois, avant d'annuler les décisions du Conseil, le Tribunal aurait dû aussi vérifier si la décision nationale ancienne sur laquelle le Conseil de l'Union se fondait ne continuait pas à fournir une base suffisante pour justifier la désignation. Or il ne l'a pas fait.

En revanche, toujours selon l'avocat général, les décisions du Conseil de l'Union d'inscrire les Tigres Tamouls et le Hamas sur la liste des organisations terroristes de I'Union, que ces entités attaquaient, devraient être annulées pour un motif de procédure: en effet, le Conseil de l'Union se serait uniquement fondé sur les éléments nouveaux, alors que ce qu'il devait faire était de montrer que les décisions anciennes étaient toujours pertinentes.

II reste à présent à attendre l'arrêt de la Cour sur ce sujet avant de revoir la pratique du Conseil lorsqu'il envisage de renouveler la désignation d'une personne ou entité sur la liste des organisations terroristes de l'Union. En effet, il semble bien ressortir de ces affaires que la pratique du Conseil devra être ajustée en ce que le Conseil s'attache aujourd'hui à démontrer que la désignation elle-même reste justifiée, alors qu'il devrait démontrer que la décision nationale à la base de la désignation demeure pertinente. II s'agit désormais de déterminer ce que cela implique. Se pourraitil que, en pratique à défaut de l'être en théorie, la nuance entre «éléments nouveaux que le Conseil aurait lui-même établi» (interdits) et "démonstration que les décisions anciennes sont toujours pertinentes» (requise) ne soit pas considérable, de sorte qu'un ajustement de la motivation des décisions de renouvellement puisse suffire à assurer la légalité de ses décisions?

Quoiqu'il en soit, on retrouve, dans l'examen du régime autonome de lutte contre le terrorisme, la même obligation particulièrement exigeante de respecter les valeurs qui sous-tendent la construction européenne qui était apparue dans l'examen du régime onusien de lutte contre le terrorisme.

Comme ces lignes l'ont montré, pour l'Union Européenne, la lutte contre le terrorisme est une question existentielle. Elle l'est d'abord parce que, comme l'année 
2016 l'a tragiquement illustré, le niveau de menace que le terrorisme fait peser sur la cohésion même de nos sociétés a rarement été aussi élevé. Elle l'est ensuite parce qu'elle peut rendre indispensable le renforcement des contrôles, notamment aux frontières, ou encore la volonté de chaque État membre de procéder à ses propres évaluations sans nécessairement tenir compte de celles des autres États membres dans un esprit de confiance mutuelle. Ainsi, c'est le cœur même de ce qui constitue ce formidable projet de paix et de circulation sur le territoire européen qu'est l'Union Européenne qui pourrait être, peu à peu, désactivé. La lutte contre le terrorisme est une question existentielle pour l'Union, enfin, parce que la vocation du traité de l'Union à être autre chose qu'un simple traité, mais bien une charte constitutionnelle de base, repose en grande partie sur la capacité de l'Union à articuler efficacité de l'action publique et défense de ses valeurs fondamentales. Telle est bien la lourde tâche à laquelle la Cour s'est, à juste titre, attelée. 